\title{
Fast-FISH detection and semi-automated image analysis of numerical chromosome aberrations in hematological malignancies
}

\author{
Arif Esa ${ }^{\mathrm{a}}$, Luba Trakhtenbrot ${ }^{\mathrm{b}}$, Michael Hausmann ${ }^{\mathrm{a}}$, Joachim Rauch ${ }^{\mathrm{a}}$, Frida Brok-Simoni ${ }^{\mathrm{b}}$, \\ Gideon Rechavi ${ }^{\mathrm{b}}$, Isaac Ben-Bassat ${ }^{\mathrm{b}}$ and Christoph Cremer ${ }^{\mathrm{a}, *}$ \\ a Institute of Applied Physics, Heidelberg, F.R. Germany \\ ${ }^{\mathrm{b}}$ Institute of Hematology, The Chaim Sheba Medical Center, Tel-Hashomer, and Sackler Faculty of \\ Medicine, Tel-Aviv University, Israel
}

Received 8 October 1997

Revised 23 February 1998

Accepted 26 February 1998

\begin{abstract}
A new fluorescence in situ hybridization (FISH) technique called Fast-FISH in combination with semi-automated image analysis was applied to detect numerical aberrations of chromosomes 8 and 12 in interphase nuclei of peripheral blood lymphocytes and bone marrow cells from patients with acute myelogenous leukemia (AML) and chronic lymphocytic leukemia (CLL). Commercially available $\alpha$-satellite DNA probes specific for the centromere regions of chromosome 8 and chromosome 12, respectively, were used. After application of the Fast-FISH protocol, the microscopic images of the fluorescencelabelled cell nuclei were recorded by the true color CCD camera Kappa CF $15 \mathrm{MC}$ and evaluated quantitatively by computer analysis on a PC. These results were compared to results obtained from the same type of specimens using the same analysis system but with a standard FISH protocol. In addition, automated spot counting after both FISH techniques was compared to visual spot counting after standard FISH. A total number of about 3,000 cell nuclei was evaluated. For quantitative brightness parameters, a good correlation between standard FISH labelling and Fast-FISH was found. Automated spot counting after Fast-FISH coincided within a few percent to automated and visual spot counting after standard FISH. The examples shown indicate the reliability and reproducibility of Fast-FISH and its potential for automatized interphase cell diagnostics of numerical chromosome aberrations. Since the Fast-FISH technique requires a hybridization time as low as $1 / 20$ of established standard FISH techniques, omitting most of the time consuming working steps in the protocol, it may contribute considerably to clinical diagnostics. This may especially be interesting in cases where an accurate result is required within a few hours
\end{abstract}

Keywords: Fast-FISH, image analysis, spot counting, chromosomal aberrations, hematological malignancies

\section{Introduction}

Specific chromosomal abnormalities play an important role in the initiation and progression of neoplastic diseases. Cytogenetic analysis is an integral part of the routine evaluation of patients suffering from hematological malignancies. Structural or numerical karyotypic abnormalities of neoplastic cells [20] can be viewed as disease-associated markers providing not only diagnostic but also prognostic information. They can be employed for monitoring the remission or the relapse status of a patient and thus affect the therapy $[22,26,28,32]$.

\footnotetext{
${ }^{*}$ Correspondence address: Prof. Dr. Christoph Cremer, Institut für Angewandte Physik, Albert-Überle-Str. 3-5, D-69120 Heidelberg, F.R. Germany. Tel.: +496221 549250; Fax: +496221549262.
} 
Trisomy of chromosome 8 in acute myelogenous leukemia (AML) is associated with low remission rates and short remission duration [1]; in chronic myelogenous leukemia (CML) involved in karyotypic aberrations in addition to the Philadelphia chromosome, it indicates the development towards the terminal phase of the disease $[7,14,21,27]$. Polysomies of chromosome 8 are mostly associated with a poor prognosis, i.e., a short survival time [30,31]. In chronic lymphocytic leukemia (CLL), cases with trisomy 12 represent a subgroup with a typical morphology associated with an advanced stage of disease and worse prognosis [19]. Thus, the detection of the size of polysomal clones may be helpful to select the intensity of the therapy.

Interphase cytogenetics [9] by means of fluorescence in situ hybridization (FISH) has offered new perspectives to a large scale analysis of tumour cells $[8,15,29]$. This has provided the possibility to delineate very small chromosomal subclones and their importance in clinical oncology. The early discovery of minute abnormalities can serve as an indicator for the beginning of leukemogenesis, early relapse, minimal residual disease or therapy response, and can have an impact on clinical decisions.

Although established FISH techniques have reached a certain standard [5], especially when applying commercially available probe kits, requirements for improved automatization exist in order to obtain a more rapid, highly reproducible FISH diagnosis from bone marrow or lymphocyte cell nuclei in hemato-oncological routine use. Nearly all standard FISH techniques [18] are based on chemical (typically formamide) denaturation of probe and target DNA supported by heat treatment. This is followed by a prolonged hybridization time (overnight up to several days) and a time consuming post-hybridization and washing procedure.

The recently developed Fast-FISH technique $[6,16]$ omits chemical denaturing agents and consequently all working steps correlated in their application. The stringency of probe binding is controlled only by hybridization temperature and time. Fast-FISH has reproducibly been proven as a rapid procedure well suited for quantitative microscopy by digital computer image analysis [10-13,17].

Here, first applications of Fast-FISH to hematological specimens are reported analyzing numerical aberrations of chromosomes 8 and 12 in bone marrow and peripheral blood cell nuclei. In each case the size of the disomic, trisomic and tetrasomic clones was analyzed by three different methods: standard FISH with visual counting of hybridization signals, standard FISH with quantitative image analysis, and Fast-FISH with quantitative image analysis. The comparison between the three methods revealed a good correlation and demonstrated the application potential of Fast-FISH for semi-automated, i.e., interactive computer analysis of numerical chromosome aberrations in hematological malignancies.

\section{Materials and methods}

\subsection{Specimen}

Peripheral blood lymphocytes were collected from four patients with B-cell origin chronic lymphocytic leukemia (B-CLL). Three of them $(12-1,12-2,12-3)$ were characterized by trisomy 12; patient 12-C had no trisomy 12 (used as control). The specimens were hybridized with a chromosome 12 specific centromere probe.

Bone marrow cells were collected from patients with acute myelogenous leukemia (AML). Patient 8-1 was characterized by trisomy 8 , patient $8-2$ by trisomy and tetrasomy of chromosome 8 . No numerical aberration of chromosome 8 was seen in patient 8 -c (used as control). These specimens were hybridized with a chromosome 8 specific centromere probe. 
According to standard methods, the cells were cultured in RPMI-1640 medium with $10 \%$ fetal calf serum for 24-48 hours without mitogenes and harvested after 30 min Colcemid exposure. After incubation with $75 \mathrm{mM} \mathrm{KCl}$ at $37^{\circ} \mathrm{C}$ for 20 min the cells were fixed with methanol: acetic acid $(3: 1 ; \mathrm{v}: \mathrm{v})$.

\subsection{Standard FISH}

For standard FISH, commercially available biotinylated (Oncor, Gaithersburg, MD, USA) or digoxigenin labelled (Boehringer Mannheim, Mannheim, FRG) DNA probes specific for the centromere regions of chromosomes 8 and 12, respectively, were used according to a protocol established in the routine laboratory of the Institute of Hematology, Tel-Hashomer:

(a) Pre-hybridization treatment of specimen slides:

- air drying at $37^{\circ} \mathrm{C}$ overnight;

- RNase $(100 \mu \mathrm{g} / \mathrm{ml}$ in $2 \times \mathrm{SSC} ; \mathrm{pH} 7.0)$ treatment at $37^{\circ} \mathrm{C}$ for 1 hour;

- washing in $2 \times \mathrm{SSC}$ for $3 \mathrm{~min}$;

- dehydration by an ethanol series $(70,80,90,100 \%)$ at room temperature;

- denaturation in $70 \%$ formamide in $2 \times \mathrm{SSC}$ at $73^{\circ} \mathrm{C}$ for $3 \mathrm{~min}$;

- dehydration by an ethanol series $(70,80,90,100 \%)$ at $-20^{\circ} \mathrm{C}$;

- proteinase $\mathrm{K}(60-100 \mu \mathrm{g} / \mathrm{ml}$ in $20 \mathrm{mM}$ Tris- $\mathrm{NaCl} / 2 \mathrm{mM} \mathrm{CaCl}$; $\mathrm{pH} 7.5)$ digestion at $37^{\circ} \mathrm{C}$ for $7.5 \mathrm{~min}$;

- dehydration by an ethanol series $(70,80,90,100 \%)$.

(b) Pre-hybridization preparation of the probe:

- mixing of $1.5 \mu \mathrm{l}$ probe/slide with $30 \mu \mathrm{l}$ Oncor Hybridizol VI (containing 65\% formamide) at $37^{\circ} \mathrm{C}$ for $5 \mathrm{~min}$;

- heat denaturation at $70^{\circ} \mathrm{C}$ for $5 \mathrm{~min}$.

(c) Hybridization:

- adding denatured probe solution on prewarmed specimen slides at $37^{\circ} \mathrm{C}$;

- incubation of slides covered with a cover slip in a humidified chamber at $37^{\circ} \mathrm{C}$ for $16-18 \mathrm{~h}$.

(d) Post-hybridization washing:

- three times in $50 \%$ formamide $/ 2 \times \mathrm{SSC}(\mathrm{pH} 7.0)$ at $45^{\circ} \mathrm{C}$ for 3 min each;

- twice in $2 \times \mathrm{SSC}(\mathrm{pH} 7.0)$ at $37^{\circ} \mathrm{C}$ for 4 min each.

(e) Detection of labelling sites:

According to the manufacturer's protocol, digoxigenin labelling was visualized by anti-digoxigenin-FITC followed by an amplification with a rabbit-anti-sheep and FITC-anti-rabbit antibody system. Biotinylated probes were visualized by FITC-avidin followed by an amplification with a FITC-anti-avidin antibody system. The unlabelled DNA of the cell nuclei was counterstained with propidium iodide $(0.5 \mu \mathrm{g} / \mathrm{ml})$.

\subsection{Fast-FISH}

For Fast-FISH, commercially available digoxigenin labelled $\alpha$-satellite DNA probes (Boehringer Mannheim, Mannheim, FRG) specific for chromosomes 8 and 12, respectively, were used according to the protocol recently published [10] but with minor modifications: 
(a) Pre-hybridization treatment of specimen slides:

- not necessary.

(b) Pre-hybridization preparation of the probe:

- mixing of $20 \mathrm{ng}$ DNA probe (=1 $\mu \mathrm{l}$ DNA probe solution) with $5 \mu \mathrm{l}$ Tris- $\mathrm{HCl}(10 \mathrm{mmol} / \mathrm{l}) / \mathrm{NaCi}$ $(30 \mathrm{mmol} / \mathrm{l})$ and $2 \mu \mathrm{l} \mathrm{NaCl}(800 \mathrm{mmol} / \mathrm{l})$ at $\mathrm{pH} 8.0$;

- diluting in deionized $\mathrm{H}_{2} \mathrm{O}$ up to a final volume of $10 \mu$ l.

(c) Hybridization:

- pipetting of hybridization mix on specimen slides;

- denaturing of both, probe and target, at $95^{\circ} \mathrm{C}$ for $5 \mathrm{~min}$ in a closed stainless steel chamber after sealing the slides with a cover slip by rubber cement (Fixogum, Marabu, Tamm, FRG);

- hybridization in the reaction chamber at $74^{\circ} \mathrm{C}$ for $15 \mathrm{~min}$. (In order to optimize the hybridization conditions various studies at $60,68,74^{\circ} \mathrm{C}$, and $15,30,60,120$ min were performed.)

(d) Post-hybridization washing:

- once in $0.9 \% \mathrm{NaCl} / 0.2 \%$ Tween 20 at room temperature for $5 \mathrm{~min}$.

(e) Detection of labelling sites:

- adding of $60 \mu \mathrm{l}$ anti-digoxigenin-fluorescein Fab fragments (=200 ng/ml stock solution in $1 \times$ PBS; Boehringer Mannheim, Mannheim, FRG);

- incubation at $37^{\circ} \mathrm{C}$ for $30 \mathrm{~min}$;

- single washing in $0.9 \% \mathrm{NaCl} / 0.2 \%$ Tween 20 at room temperature for $2 \mathrm{~min}$;

- mounting in Vectashield antifade medium and counterstaining with propidium iodide $(0.2 \mu \mathrm{g} /$ $\mathrm{ml})$.

\subsection{Microscopy and image analysis}

\subsubsection{Visual analysis}

Each specimen slide was analyzed twice using an Olympus BH 2 fluorescence light microscope equipped with a PlanApo objective $100 \times / 1.4$ oil, appropriate spectral filter, and a $100 \mathrm{~W}$ mercury arc lamp. Between 200 and 335 nuclei were evaluated per case. Cell nuclei without FISH signal were not considered for evaluation.

\subsubsection{Semi-automated analysis}

A Leica DMR fluorescence light microscope was used, equipped with a PlanApo 63×/1.4 oil objective and a $100 \mathrm{~W}$ mercury arc lamp. Excitation took place via a 450-490 $\mathrm{nm}$ band pass filter and emission via a $515 \mathrm{~nm}$ long pass filter. Cell nuclei carrying FISH signals were chosen by random access. Their images were recorded with the cooled true color CCD camera Kappa CF 15 MC (Kappa Meßtechnik, Gleichen, FRG) [2]. The images were transferred to a color frame grabber (ITI Vision Plus Color (CFG 512; Kappa Meßtechnik)) and digitized. Controlling of the camera and evaluation of the images was performed interactively using the commercially available software package OPTIMAS (BioScan, Edmonds, WA, USA) running on a PC under Windows 3.1 with the MS-DOS operating system. In this software package a specially designed program called FISHSPOT was implemented for semi-automated quantitative image analysis (see, e.g., [6]). 

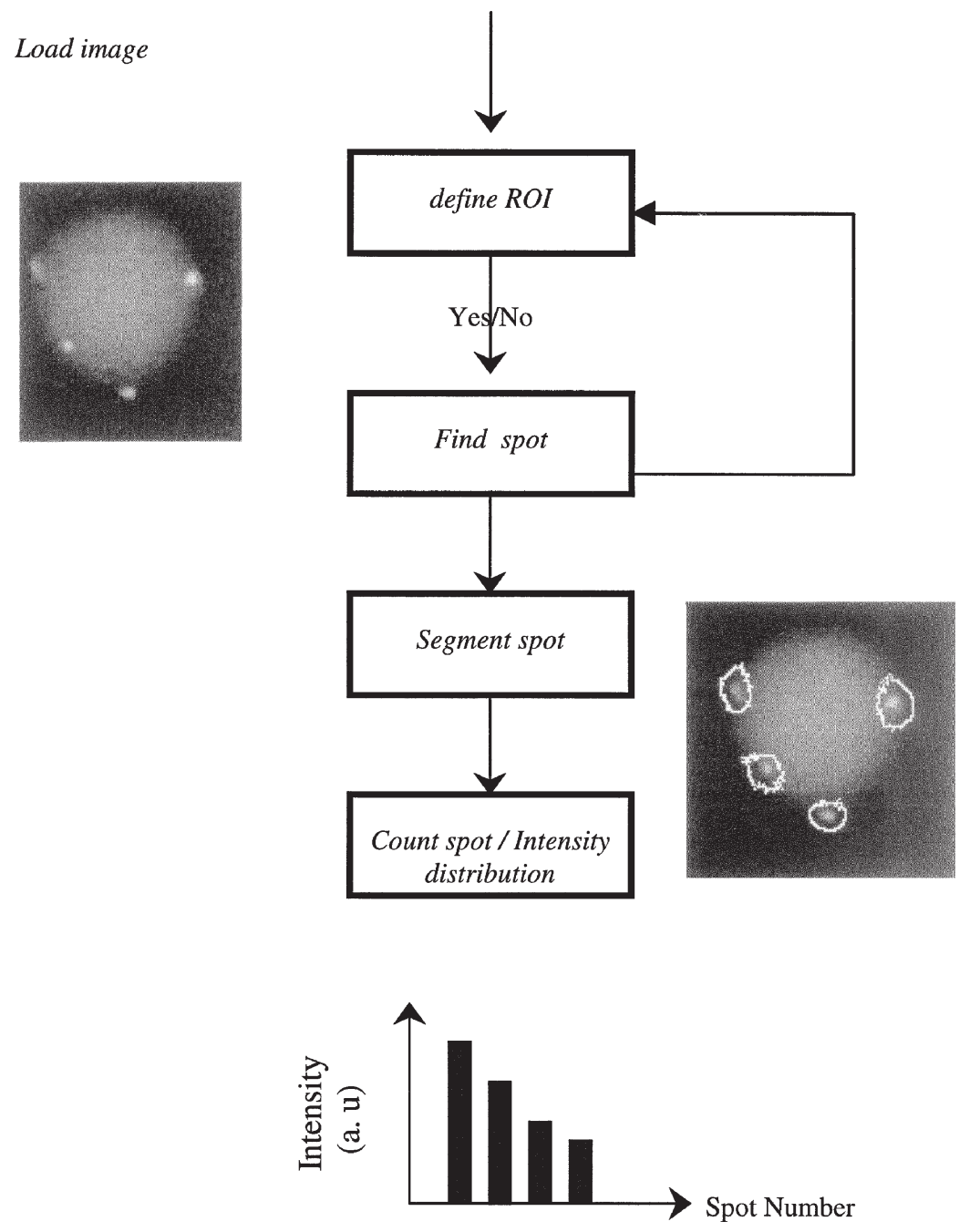

Fig. 1. Schematic description of the spot finding algorithm. The digitized raw image (left) was subjected to several analysis steps. The results are an image with segmented labelling sites (right) from which quantative values of intensity or area, for instance, can be calculated.

In FISHSPOT, the FISH signals of the labelling sites were automatically identified and segmented in the RGB-image plane corresponding to their colour (here green image plane for FITC). The user interactively selected a region of interest (ROI) on the screen. For segmentation, local intensity maxima were fully automatically determined within the ROI and the surrounding intensity distributions were analysed. Along the points comparable to the local maxima in the second derivative the segmentation borderline was fixed. Besides spot counting per nucleus a pattern of quantitative spot parameters was obtained (Fig. 1).

For comparing the labelling quality of the applied FISH techniques, three different intensity parameters were used: (a) mean intensity $S$ of all pixels of a spot; (b) maximum intensity $S_{\max }$ of the pixels of a spot; (c) integrated intensity of a spot $S \times F$ ( $F=$ area of the spot in pixels). These spot parameters were further processed by a standard commercially available spreadsheet program and graphically visualized. 
Furthermore, scan lines with a width equivalent to 10 pixels were interactively drawn through the labelling spots in individual images. Profiles representing the intensity distribution in the green image plane along the scan line were obtained to get an information about image contrast. Per case and FISH technique, between 102 and 258 cell nuclei were evaluated.

\section{Results}

Standard FISH and Fast-FISH were compared according to signal quality (image contrast and image intensity) and signal number (number of labelling sites) by semi-automated image analysis for all patients and controls. The signal number representing the detected numerical chromosome aberrations was correlated to results obtained by routine visual spot counting to get an estimate of the error rate and its tolerability.

Figure 2(A) shows typical digitized images of cell nuclei from 8-2, 12-1 and 12-C after Fast-FISH in comparison to cell nuclei after standard FISH. In Fig. 2(B) the corresponding intensity profiles through the labelling sites are shown. The figures indicate a several-fold contrast between the intensity of the labelling sites and the registered fluorescence background along the scan line. No FISH technique dependent differences in the general contrast behaviour was found. The figures also support the visual impression that considerable differences in the FISH efficiency expressed by FISH signal brightness were not found for bone marrow and peripheral blood cells (e.g., 8-2 compared with 12-1).

Since the aim of this study was not only to test the feasibility of Fast-FISH but also the suitability of this technique to semi-automated microscopy, three image parameters (see Section 2) were tested. In all cases, the signal parameters $S, S_{\max }, S \times F$ showed compatible values for both FISH techniques and all labelling sites. In some experiments the brightness values of Fast-FISH signals appeared to be slightly increased compared to standard FISH. In Fig. 3 the results are shown for 8-2, the patient with a certain amount of tetrasomic cells. The figure shows the mean values and standard deviation of the four possible spots averaged over 132 (standard FISH) or 129 (Fast-FISH) cell nuclei, respectively. For all three parameters, the values vary over a certain range. However, $S \times F$ depending on the spot area segmentation shows the largest variability. Therefore, $S$ or $S_{\max }$ were used as the preferred parameters for automated image analysis and spot classification.

To further test the diagnostic variability of the applied techniques, spot counting was performed and the percentage frequencies of cell nuclei with one, two, three and four spots were determined (Table 1). From the control cases $12-\mathrm{C}$ and $8-\mathrm{C}$, where the expected number of labelling sites was 2 , an error rate due to insufficient hybridization, optical fusion (the image is a 2-dimensional projection of a 3-dimensional object) or reduced stringency may be estimated. About $94 \%$ of two spot cell nuclei were found by visual analysis, $90 \%$ by (semi-)automated analysis after standard FISH, and about $87 \%$ by (semi-)automated analysis after Fast-FISH. For the latter it should be considered that a lower number of cell nuclei was analyzed.

The results obtained by the three analytic techniques were in good correlation. In case 12-3, less than $30 \%$ of the cell nuclei were trisomic and about $2 / 3$ of all cell nuclei showed the normal karyotype (two chromosomes 12). In case $12-1$ about $40 \%$ and in case $12-2$ more than $50 \%$ cell nuclei with a trisomy were found. Compared to the control, all values confirmed a significant increase of trisomic cell nuclei. In case $8-1$, about $19 \%$ of the cell nuclei had a trisomy 8 , about $70 \%$ showed a normal karyotype. In case $8-2$, about $20 \%$ of the cells had a tetrasomy 8 . This means that more than $50 \%$ of the analyzed cell nuclei were already aberrant. 
Fast- FISH

A

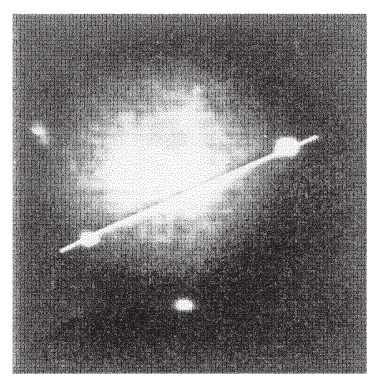

8-2

B

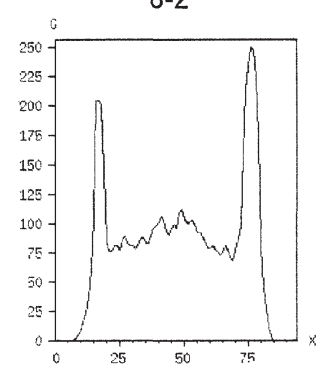

A

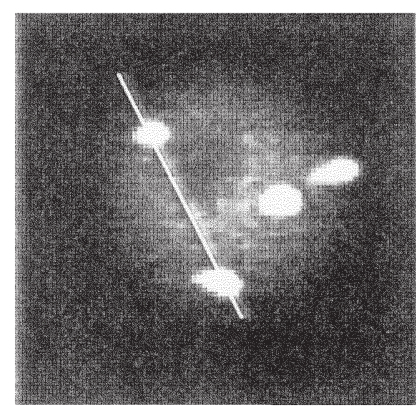

8-2

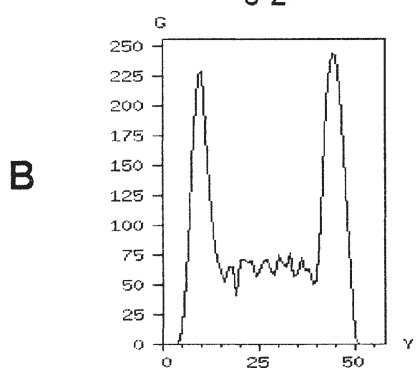

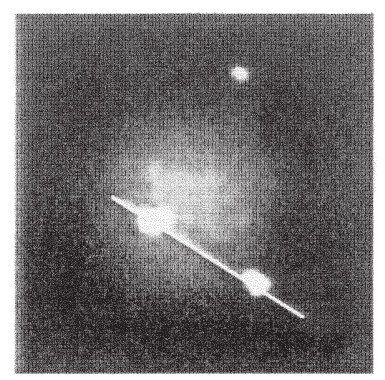

12-1

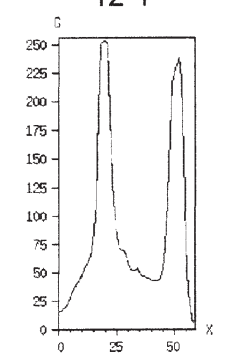

Standard FISH

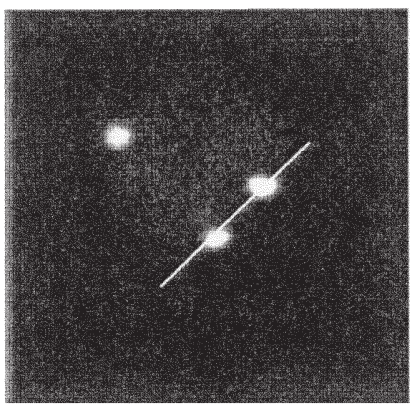

$12-1$

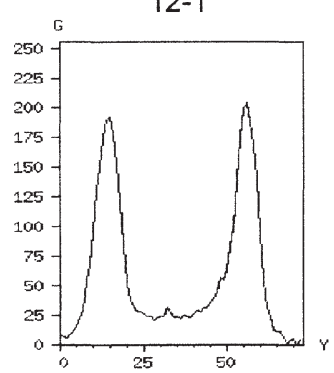

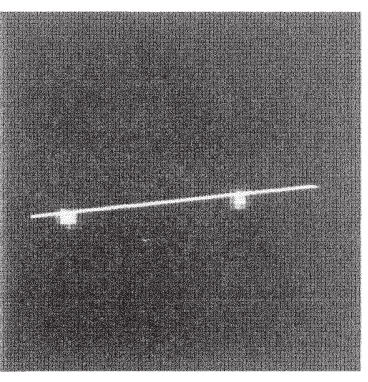

$12-C$
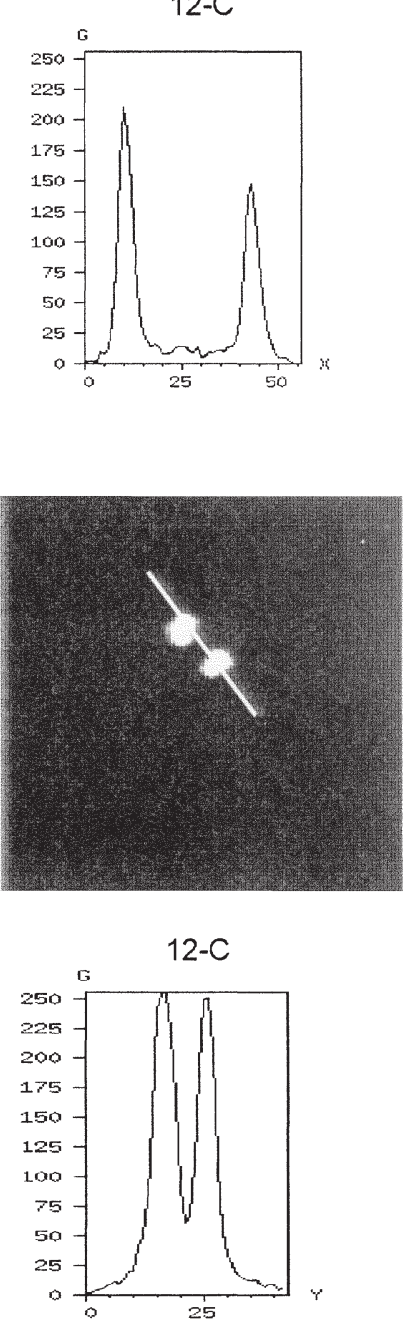

Fig. 2. (A) Digitized images of typical cell nuclei of the patients 8-2 (AML with tetrasomy 8), 12-1 (B-CLL with trisomy 12), and 12-C (no numerical aberration of chromosome 12) after Fast-FISH and standard FISH. (B) Intensity profiles from the green image plane along the scan lines indicated in (A). The ordinate shows gray values in arb. units. On the abscissa the length of the scan in arb. units is shown. 


\section{Standard FISH}
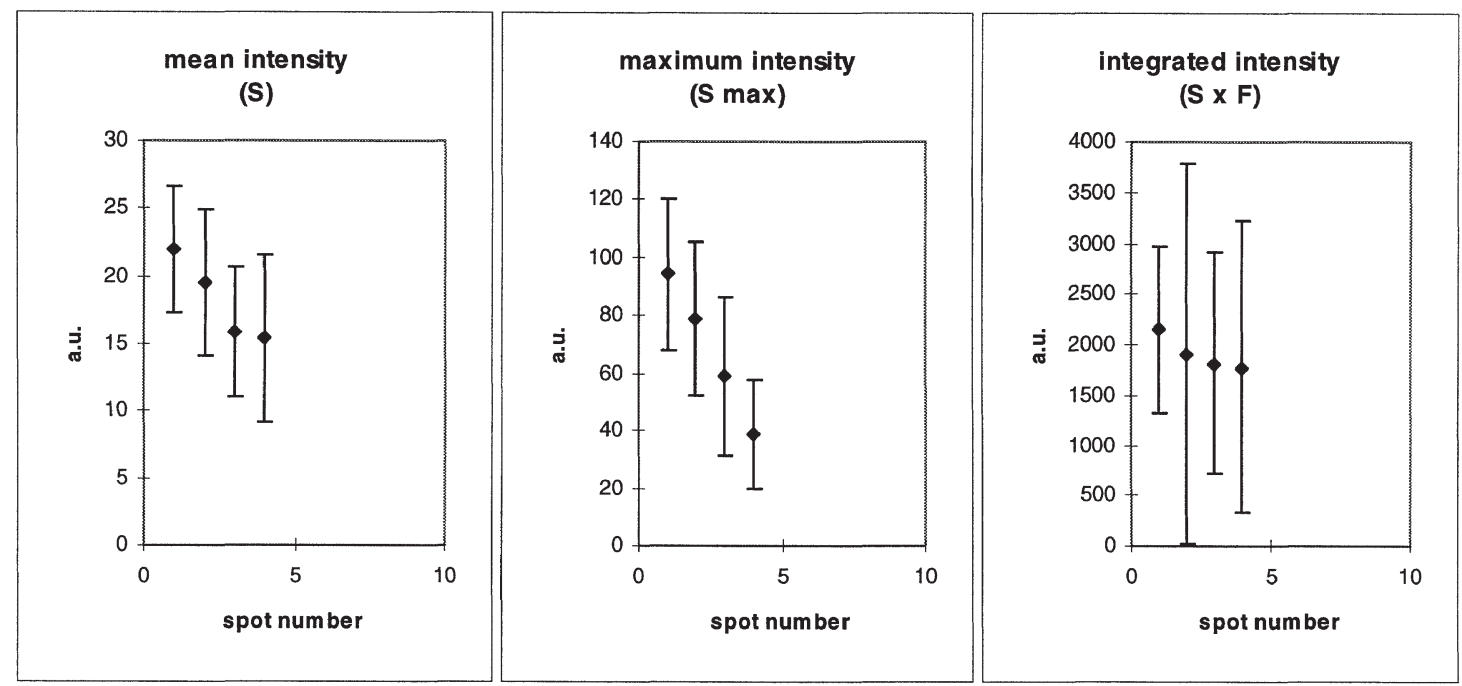

\section{Fast FISH}
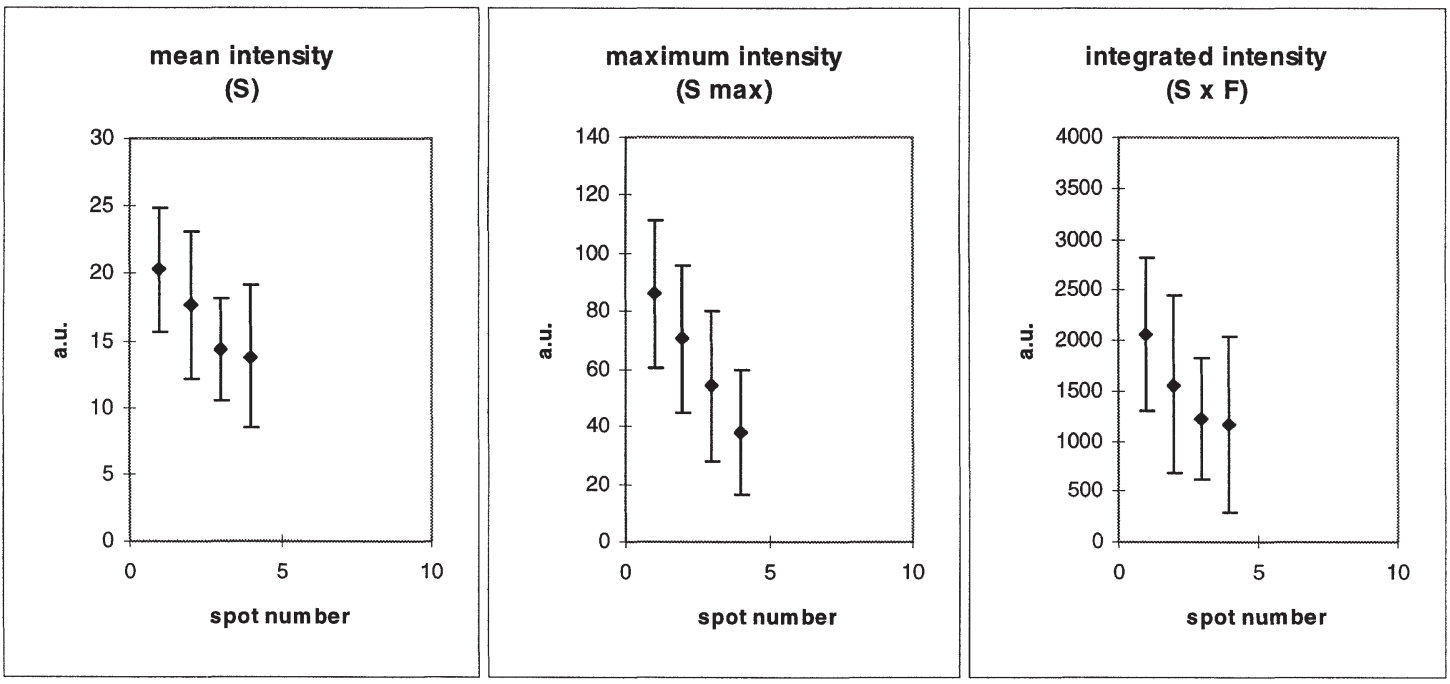

Fig. 3. The classification parameters of labelling sites shown for standard and Fast-FISH for patient 8-2 (AML with tetrasomy 8). 132 cell nuclei (standard FISH) or 129 (Fast-FISH), respectively, were evaluated. According to their brightness the spots of each nucleus were classified. The brightest, second brightest, third brightest, etc., spots were listed (abscissa) and averaged. The points represent the mean values with their standard deviation.

Figure 4 shows two correlation curves comparing visual with semi-automated analysis for standard FISH, and standard FISH with Fast-FISH for semi-automated analysis. Both correlation curves showed a highly proportional behaviour according to $R^{2}$ (= square of the Pearson correlation coefficient). In Fig. 4(a) comparing Fast-FISH with standard FISH, $R^{2}$ was 0.96 corresponding to $98 \%$ of the variance depending on linear regression effects. In Fig. 4(b) comparing semi-automated to visual evaluation, $R^{2}$ was 0.95 corresponding to $97 \%$ of the variance depending on linear regression effects. These results 
Table 1

Spot counting in cell nuclei from the same specimen after three techniques: (A) visual counting after standard FISH; (B) semiautomated counting after standard FISH; (C) semi-automated counting after Fast-FISH. For all cases, the absolute number of cell nuclei $N$ and the percentage frequencies of cell nuclei with $1,2,3,4$ hybridization spots are given. Cells without any hybridization signals were not considered. For the control cases 12-C and 8-C, always two spot cell nuclei were expected. Thus the measured percentages of one and three spot cell nuclei can be used as a measure for the methodological error rates due to insufficient hybridization, optical fusion and reduced stringency. (See also Fig. 4)

\begin{tabular}{|c|c|c|c|c|c|c|c|c|c|c|c|c|c|c|c|}
\hline \multirow[t]{2}{*}{ Case } & \multicolumn{5}{|c|}{ A } & \multicolumn{5}{|c|}{ B } & \multicolumn{5}{|c|}{$\mathrm{C}$} \\
\hline & $N$ & 1 & 2 & 3 & 4 & $N$ & 1 & 2 & 3 & 4 & $N$ & 1 & 2 & 3 & 4 \\
\hline $12-1$ & 210 & 3.1 & 56.4 & 40.4 & 0 & 149 & 6.7 & 54.4 & 38.9 & 0 & 102 & 16.7 & 38.2 & 45.1 & 0 \\
\hline $12-2$ & 280 & 5.2 & 44.3 & 50.5 & 0 & 237 & 6.3 & 31.2 & 62.4 & 0 & 158 & 5.1 & 42.4 & 52.5 & 0 \\
\hline $12-3$ & 200 & 3.2 & 74.2 & 22.5 & 0 & 152 & 5.9 & 65.8 & 28.3 & 0 & 140 & 8.6 & 64.3 & 27.1 & 0 \\
\hline $12-\mathrm{C}$ & 250 & 5.0 & 94.5 & 0.5 & 0 & 238 & 8.4 & 89.5 & 2.1 & 0 & 139 & 12.9 & 84.9 & 2.2 & 0 \\
\hline 8-1 & 218 & 13.2 & 64.8 & 19.8 & 0 & 202 & 5.0 & 76.2 & 18.8 & 0 & 221 & 5.9 & 74.2 & 19.9 & 0 \\
\hline 8-2 & 335 & 9.5 & 41.8 & 36.7 & 11.3 & 132 & 5.3 & 38.6 & 33.3 & 22.7 & 129 & 8.5 & 34.9 & 32.6 & 24.0 \\
\hline 8-C & 250 & 5.1 & 93.2 & 1.7 & 0 & 216 & 4.2 & 91.7 & 4.2 & 0 & 108 & 8.3 & 89.8 & 2.8 & 0 \\
\hline
\end{tabular}

$\chi^{2}(\mathrm{~B} ; \mathrm{C})=37.69>\chi_{15 ; 0.01}^{2}, \chi^{2}(\mathrm{~A} ; \mathrm{B})=48.57>\chi_{15 ; 0.01}^{2}$.

indicate that the three analytic techniques tested can be applied equally for the analysis of numerical chromosome aberrations. This was supported by the application of a $\chi^{2}$-test. For Fig. 4(a), $\chi^{2}=37.69$, and for Fig. 4(b), $\chi^{2}=48.57$ were found which supports the equality of the compared techniques on the $0.1 \%$ significance-level.

\section{Discussion}

The aim of this study was to test the suitability of Fast-FISH in combination with semi-automated image microscopy for the analysis of numerical chromosome aberrations in interphase cell nuclei of bone marrow and peripheral blood cells of hemato-oncological patients. For this purpose, a comparison to standard FISH under the same image analysis procedure and standard FISH with routine visual evaluation was performed.

The major advantage of Fast-FISH is that the number of preparative steps in the protocol is extremely reduced. This reduces the time necessary for the specimen preparation for microscopy to at least $1 / 20$ of the standard technique. Using several probes simultaneously with different colors (L. Schüßler, M. Durm, H. Münch, M. Hausmann, C. Cremer, unpublished results) a further acceleration may be obtained, since this approach may allow the simultaneous detection of different numerical aberrations by means of a true color CCD camera in combination with an appropriate multi-band pass filter set. Although so-called "low-stringency" conditions were used with respect to the ingredients of the hybridization buffer and the washing procedure (omitting formamide or other chaotropic agents), the stringency can be controlled by the physical parameters hybridization temperature and time [17]. Furthermore, the quantitative evaluation of the hybridization spots obtained with repetitive $\alpha$-satellite probes for chromosomes 8 and 12 showed that no considerable differences in the relative brightness were found between the two FISH protocols.

Since single image parameter as $S$ or $S_{\max }$ can be used to classify the labelling spots, the semiautomated procedure applied here may be used for an easy standardization evaluation protocol. This is supported by the excellent correlation between automated spot counting after Fast-FISH and visual spot counting after standard FISH. The results of all three compared analysis techniques coincided signifi- 


\section{correlation between Fast FISH and Standard FISH}

(automated counting)

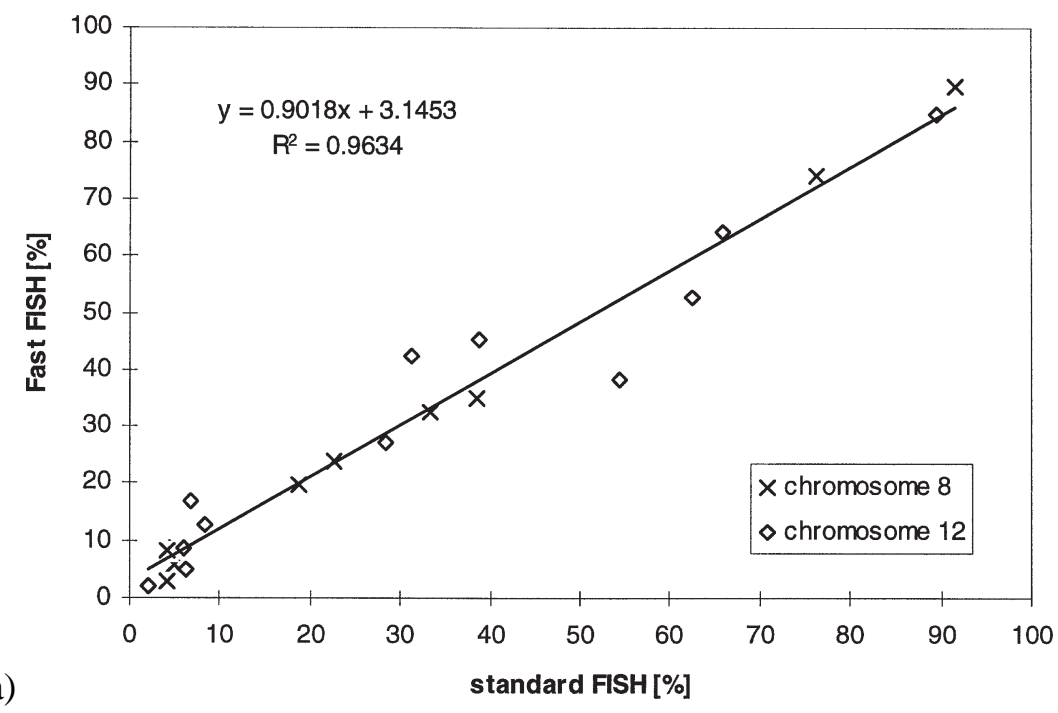

correlation between automated counting and visual counting after standard FISH

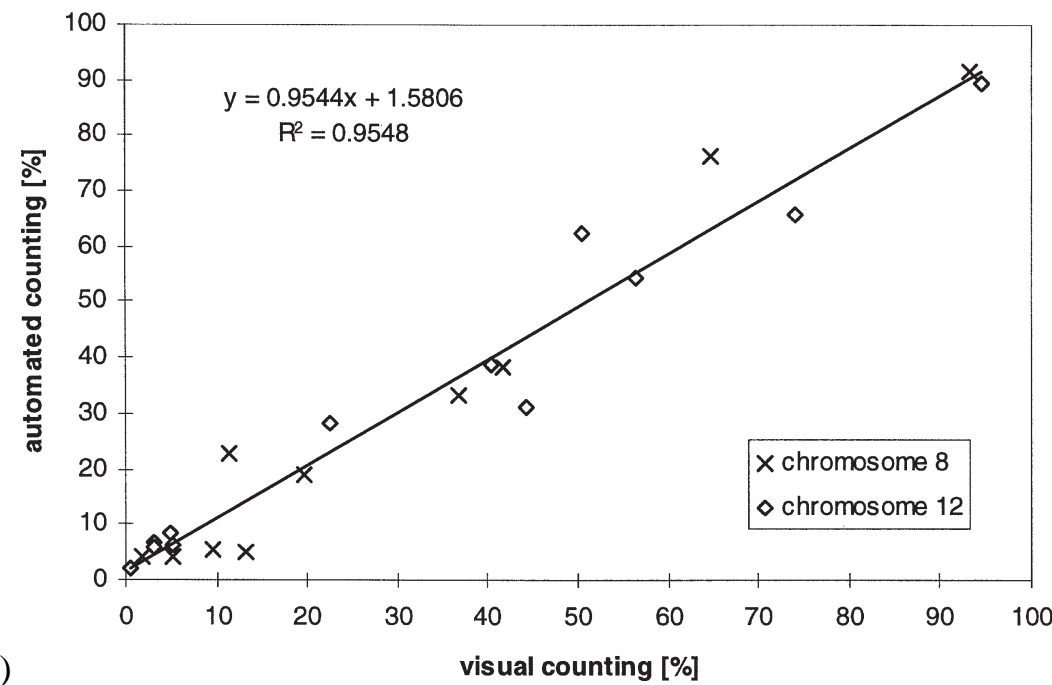

Fig. 4. Correlation of the percentage frequencies from Table 1. (a) Correlation of the values of column $\mathrm{C}$ to the values of column B. (b) Correlation of the values of column B to the values of column A. In both cases the automatically calculated fit curve $(y=a x+b)$ is given with their confidence intervals $R^{2}$ (= square of Pearson correlation coefficient). A $\chi^{2}$-test was applied. This revealed that the compared techniques give the same results on the $0.1 \%$ significance level (see Table 1 ).

cantly. Such a result has to be seen under the aspect that intercellular as well as intracellular variations in FISH labelling are known for both standard as well as Fast-FISH (see, e.g., [6,23]).

An additional influence on the results may be due to "optical fusion". The conventional epifluorescence microscope image is always a 2-dimensional projection of a 3 -dimensional object. This is especially true 
for the camera system used. In case of the visual analysis, a skilled person usually focuses slightly through the object. This may support a certain depth discrimination and perhaps be the reason for the differences in the spot counting results of the controls 8-C and 12-C. This may, for instance, be overcome by the analysis of a higher number of cell nuclei or by axial tomography [3]. This technique works with glass fibers on which the cell nuclei are fixed so that they can always be rotated into an optimal perspective [4].

The Fast-FISH technique in combination with the described semi-automated image acquisition and analysis device has the potential to become a tool in clinical diagnostics. Hundreds of cell nuclei can be easily evaluated in a few hours including the specimen hybridization. So far, the image analysis system used here requires an interactive operation. However, a recently developed fully automatized system including automated allocation of cell nuclei, automated image focussing and acquisition allows the examination of about 500 cells in approximately $15 \mathrm{~min}$ [24,25]. This system works with two fluorescent dyes, one for the hybridization spots and one for the counterstain of the entire cell nucleus. The user is no longer forced to look into a microscope for many exhausting hours since one can interactivly verify or correct the results from the monitor display. The combination of commercially available DNA probes, a fast hybridization procedure with only a few protocol steps, and digital image microscopy can be seen as a step towards higher reproducibility. The user will select any given combination of quantitative spot parameters. Together with several kinds of graphical visualization of the results, an appropriate data representation containing an optimum of diagnostic information appears to be possible.

\section{Acknowledgements}

The authors thank Klaus Aldinger, Ninneta Cohen, Dr. Markus Durm, and Dr. Frank-Martin Haar for their support and many stimulating discussions. The financial support of the Deutsche Forschungsgemeinschaft is gratefully acknowledged. A. Esa receives a scholarship of the Friedrich-Ebert-Stiftung, Bonn.

\section{References}

[1] C.D. Bloomfield, D.F. Hoeltzer and C.A. Schiffer, Acute leukemia: Recent advances in management, in: Hematology: The Education Program of the American Society of Hematology, 33th Ann. Meeting, 1991, pp. 15-22.

[2] H. Bornfleth, K. Aldinger, M. Hausmann, A. Jauch and C. Cremer, CGH imaging by the one chip true color CCD camera Kappa CF15MC, Cytometry 24 (1996), 1-13.

[3] J. Bradl, M. Hausmann, B. Schneider, B. Rinke and C. Cremer, A versatile $2 \pi$-tilting device for fluorescence microscopes, J. Microsc. 176 (1994), 211-221.

[4] J. Bradl, B. Rinke, B. Schneider, P. Edelmann, H. Krieger, M. Hausmann and C. Cremer, Resolution improvement in 3D fluorescence microscopy by object tilting, Microsc. Anal. 44(11) (1996), 9-11.

[5] N.P. Carter, Fluorescence in situ hybridization - state of the art, BioImaging 4 (1997), 41-51.

[6] D. Celeda, K. Aldinger, F.M. Haar, M. Hausmann, M. Durm, H. Ludwig and C. Cremer, Rapid fluorescence in situ hybridization with repetitive DNA probes: Quantification by digital image analysis, Cytometry 17 (1994), 13-25.

[7] F. Cervantes, M. Rozman, J. Rosell, A. Urbano-Ispizua, E. Montserrat and C. Rozman, A study of prognostic factors in blast crisis of Philadelphia chromosome-positive chronic myelogenous leukemia, Br. J. Haematol. 76 (1990), 27-32.

[8] C. Cremer and T. Cremer, Analysis of chromosomes in molecular tumor and radiation cytogenetics: approaches, applications, perspectives, Eur. J. Histochem. 36 (1992), 15-25.

[9] T. Cremer, J. Landegent, A. Brückner, H.P. Scholl, M. Schardin, H.D. Hager, P. Devilee and M. van der Ploeg, Detection of chromosome aberrations in the human interphase nucleus by visualization of specific target DNAs with radioactive and non-radiactive in situ hybridization techniques: diagnosis of trisomy 18 with probe L1.84, Hum. Genet. 74 (1986), $346-352$. 
[10] M. Durm, F.M. Haar, M. Hausmann, H. Ludwig and C. Cremer, Optimization of fast-fluorescence in situ hybridization with repetitive alpha-satellite probes, Z. Naturforsch. (J. Biosci.) 51c (1996), 253-261.

[11] M. Durm, M. Hausmann, K. Aldinger, H. Ludwig and C. Cremer, Painting of human chromosome 8 in fifteen minutes, Z. Naturforsch. (J. Biosci.) 51c (1996), 435-439.

[12] M. Durm, F.M. Haar, M. Hausmann, H. Ludwig and C. Cremer, Optimized Fast-FISH with $\alpha$-satellite probes: Acceleration by microwave activation, Braz. J. Med. Biol. Res. 30 (1997), 15-23.

[13] M. Durm, F.M. Haar, M. Hausmann, H. Ludwig and C. Cremer, Non-enzymatic, low temperature in situ hybridization of human chromosomes with a repetitive $\alpha$-satellite probe, Z. Naturforsch. (J. Biosci.) 52c (1997), 82-88.

[14] G. Fugazza, R. Bruzzone, A.M. Dejana, F. Patrone and M. Sessarego, Trisomy 8 detection in $\mathrm{Ph}^{+}$CML patients using conventional cytogenetic and interphase fluorescence in situ hybridization techniques, Cancer Genet. Cytogenet. 72 (1994), 24-27.

[15] J.W. Gray, D. Pinkel and J.W. Brown, Fluorescence in situ hybridization in cancer and radiation biology, Rad. Res. 137 (1994), 275-289.

[16] F.M. Haar, M. Durm, K. Aldinger, D. Celeda, M. Hausmann, H. Ludwig and C. Cremer, A rapid FISH technique for quantitative microscopy, Biotechniques 17 (1994), 346-353.

[17] F.M. Haar, M. Durm, M. Hausmann, H. Ludwig and C. Cremer, Optimization of Fast-FISH for $\alpha$-satellite DNA probes, J. Biochem. Biophys. Meth. 33 (1996), 43-54.

[18] P. Lichter and T. Cremer, Chromosome analysis by non-isotopic in situ hybridization, in: Human Cytogenetics: A Practical Approach, Vol. I, D.E. Rooney and B.H. Czepulkowski, eds, IRL Press, Oxford, 1992, pp. 157-192.

[19] E. Matutes, D. Oscier, J. Garcia-Marco, J. Ellis, A. Copplestone, R. Gillingham, T. Hamblin, D. Lens, G.J. Swansbury and D. Catovsky, Trisomy 12 defines a group of CLL with atypical morphology: correlation between cytogenetic, clinical and laboratory features in 544 patients, Br. J. Hematol. 92 (1995), 382-388.

[20] F. Mitelman, Catalog of Chromosome Aberrations in Cancer, 4th edn, Alan R. Liss, New York, 1991.

[21] F. Mitelman, The cytogenentic scenario of chronic myeloid leukemia, Leuk. Lymphoma 11 (1993), 11-15.

[22] F. Naem, R.A. Gatti and J.J. Yunis, Recent advances in diagnosis and classification of leukemias and lymphomas, Disease Markers 8 (1990), 231-264.

[23] P.M. Nederlof, S. van der Flier, N.P. Verwoerd, J. Vrolijk, A.K. Raap and H.J. Tanke, Quantification of fluorescence in situ hybridization signal by image cytometry, Cytometry 13 (1992), 846-852.

[24] H. Netten, L.J. van Vliet, H. Vrolijk, W.C.R. Sloos, H.J. Tanke and I.T. Young, Fluorescent dot counting in interphase cell nuclei, BioImaging 4 (1996), 93-106.

[25] H. Netten, I.T. Young, L.J. van Vliet, H.J. Tanke, H. Vrolijk and W.C.R. Sloos, FISH and chips: Automation of fluorescent dot counting in interphase cell nuclei, Cytometry 28 (1997), 1-10.

[26] T.H. Rabbits, Chromosomal translocations in human cancer, Nature 372 (1994), 143-149.

[27] A.A. Rojas, P.L. Roland, L. del Villar, N. Herrera and A. Herrera, Significance of chromosome changes in chronic myeloid leukemia, Invest. Clin. 34 (1993), 75-83.

[28] A.A. Sandberg, The Chromosomes in Human Cancer and Leukemia, 2nd edn, Elsevier, New York, 1990.

[29] D.C. Tkachuk, D. Pinkel, W.L. Kuo, H.U. Weier and J.W. Gray, Clinical applications of fluorescence in situ hybridization, Genet. Anal. Technol. Appl. 8 (1991), 67-74.

[30] L. Trakhtenbrot, E. Rosner, N. Gipsh, S. Berman, O. Sofer, F. Brok-Simoni, G. Rechavi and I. Ben-Bassat, Hexasomy of chromosome 8 and trisomy of chromosome 11 characterize two karyotypically independent clones in a case of acute non-lymphocytic leukemia, Cancer Genet. Cytogenet. 85 (1995), 1-4.

[31] U. Trautmann, M. Gramatzki, M. Krauss, A. Friz, T. Liehr and E. Gebhart, Tetrasomy 8 as a clonal anomaly in myeloid neoplasias, Cancer Genet. Cytogenet. 72 (1994), 101-104.

[32] J.J. Yunis, Genes and chromosomes in the pathogenesis and prognosis of human cancers, Adv. Pathol. 2 (1989), 147-188. 


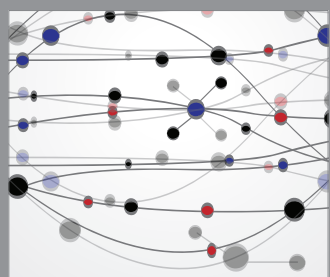

The Scientific World Journal
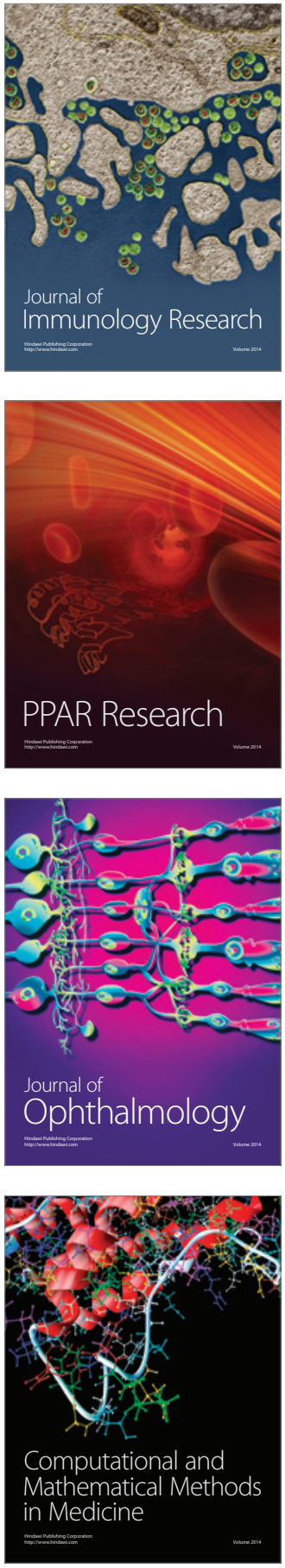

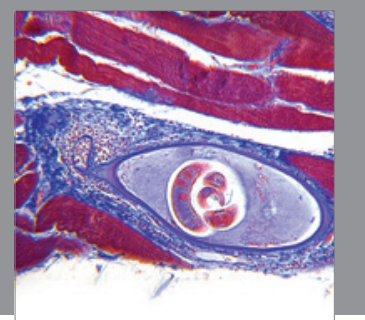

Gastroenterology

Research and Practice
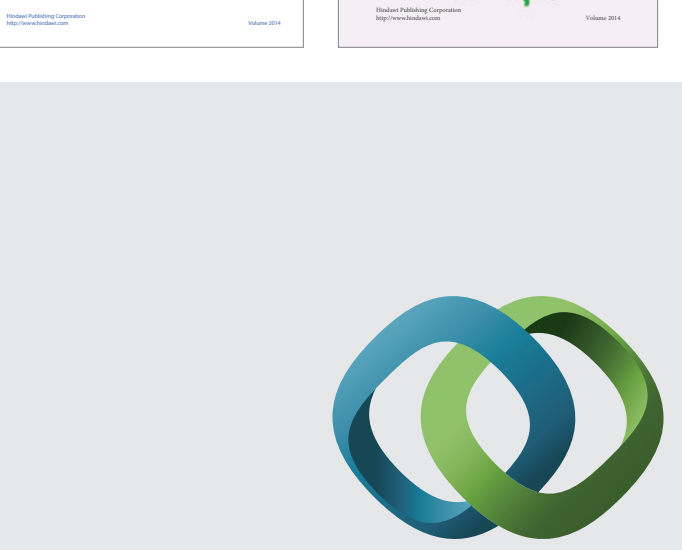

\section{Hindawi}

Submit your manuscripts at

http://www.hindawi.com
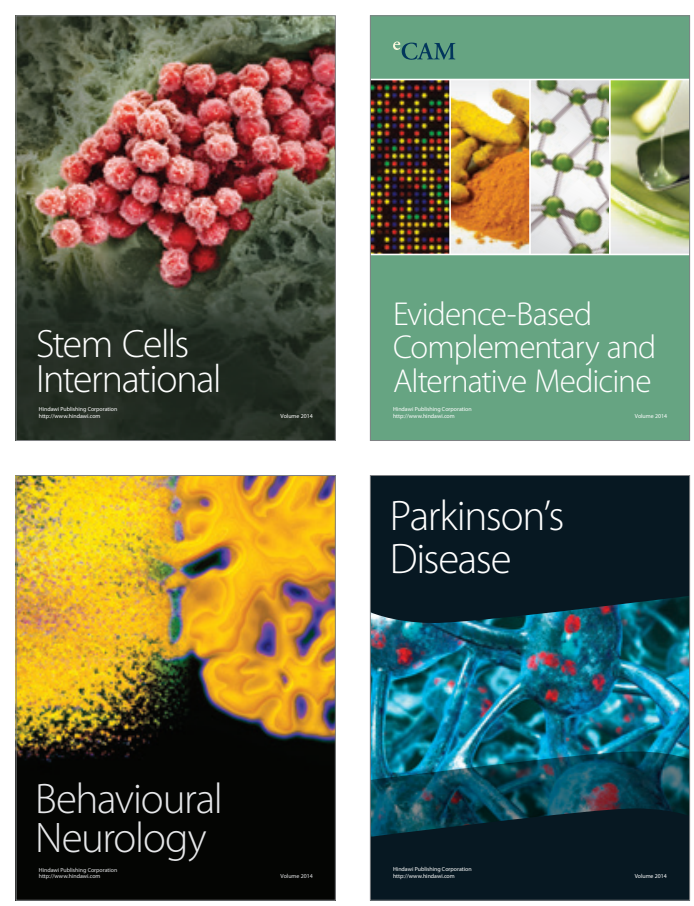

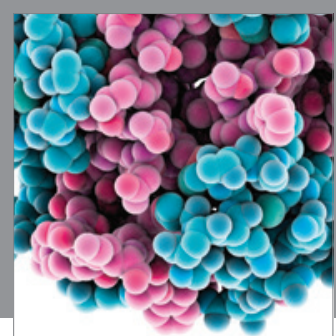

Journal of
Diabetes Research

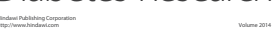

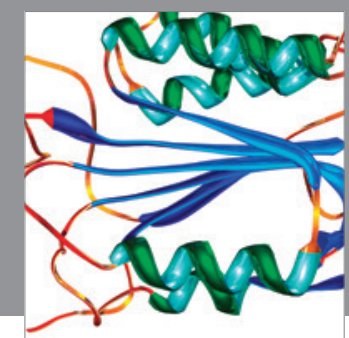

Disease Markers
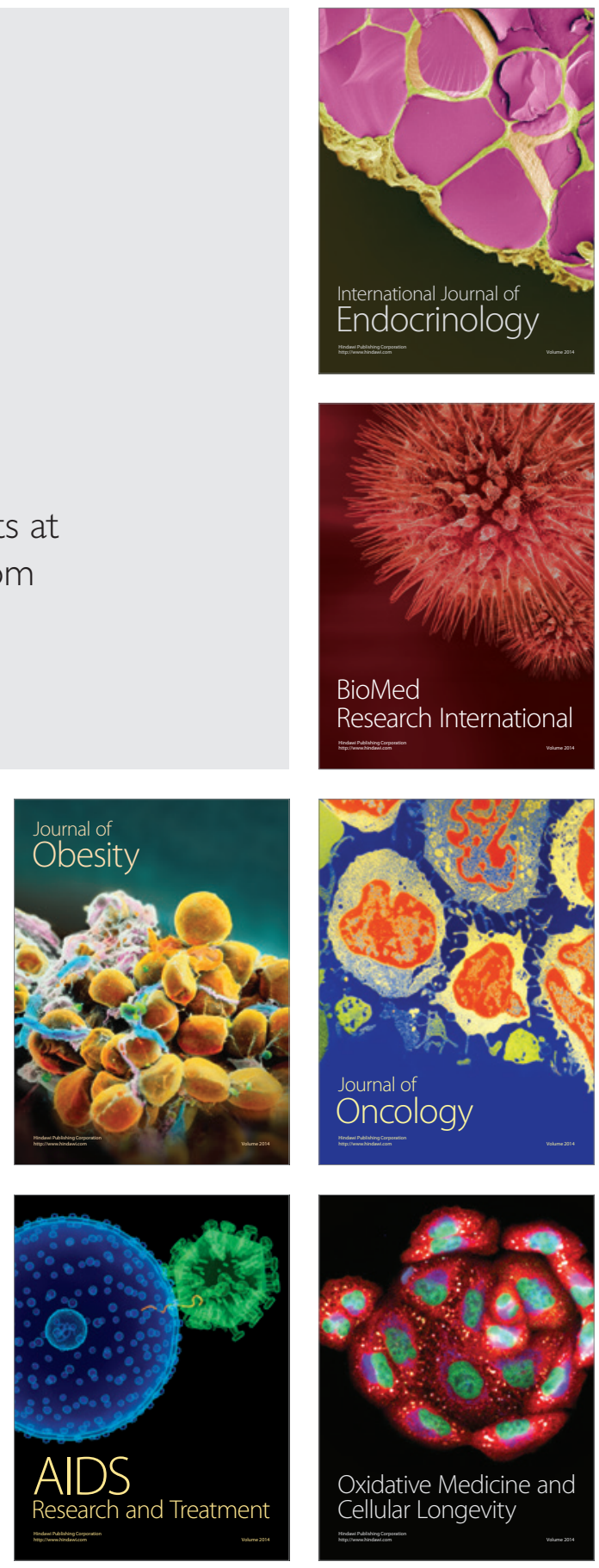